\title{
6rigersity of Glasgow
}

O'Hagan, J., Williamson, J. R. , McGill, M. and Khamis, M. (2021) Safety, Power Imbalances, Ethics and Proxy Sex: Surveying In-The-Wild

Interactions Between VR Users and Bystanders. In: 2021 IEEE

International Symposium on Mixed and Augmented Reality (ISMAR), Bari, Italy, 04-08 Oct 2021, ISBN 9781728197777

(doi:10.1109/ISMAR52148.2021.00036)

The material cannot be used for any other purpose without further permission of the publisher and is for private use only.

There may be differences between this version and the published version. You are advised to consult the publisher's version if you wish to cite from it.

http://eprints.gla.ac.uk/249305/

Deposited on 11 August 2021

Enlighten - Research publications by members of the University of

Glasgow

$\underline{\text { http://eprints.gla.ac.uk }}$ 


\title{
Safety, Power Imbalances, Ethics and Proxy Sex: Surveying In-The-Wild Interactions Between VR Users and Bystanders
}

\author{
Joseph O’Hagan* Julie R. Williamson ${ }^{\dagger} \quad$ Mark McGill $^{\ddagger} \quad$ Mohamed Khamis $^{\S}$
}

University of Glasgow

\begin{abstract}
VR users and bystanders must sometimes interact, but our understanding of these interactions - their purpose, how they are accomplished, attitudes toward them, and where they break down - is limited. This current gap inhibits research into managing or supporting these interactions, and preventing unwanted or abusive activity. We present the results of the first survey $(\mathrm{N}=100)$ that investigates stories of actual emergent in-the-wild interactions between VR users and bystanders. Our analysis indicates VR user and bystander interactions can be categorised into one of three categories: coexisting, demoing, and interrupting. We highlight common interaction patterns and impediments encountered during these interactions. Bystanders play an important role in moderating the VR user's experience, for example intervening to save the VR user from potential harm. However, our stories also suggest that the occlusive nature of VR introduces the potential for bystanders to exploit the vulnerable state of the VR user; and for the VR user to exploit the bystander for enhanced immersion, introducing significant ethical concerns.
\end{abstract}

Index Terms: Human-centered computing-Human computer interaction (HCI) - Interaction paradigms_-Virtual reality;

\section{INTRODUCTION}

VR is often used in shared, social settings, but interactions between VR users and bystanders (those physically near the VR user but who cannot directly interact with the VR user's virtual environment) in uncontrolled social settings are not well understood. Recent work has investigated a range of systems to increase a VR user's awareness of nearby bystanders $[14,34,36,40,54]$ and to facilitate interactions with them $[18,19,25,33,35,57]$. However, surprisingly little is known about VR usage within the real world settings where these systems might be used. Instead, systems are designed and tested entirely within the lab with little or no empirical evidence of the interaction being designed for actually occurring in-the-wild. Hence, an understanding of what and how interactions occur between $V R$ users and bystanders in-the-wild is missing. Addressing this gap using empirical data is important as it will allow the community to: a) better understand VR user-bystander interactions, including existing attitudes, what works, and what does not work, and b) design better approaches to facilitate these interactions and address safety and ethical concerns that may arise.

To this end, we present the results of the first survey $(\mathrm{N}=100)$ to get unbiased and unfiltered user accounts of these experiences, we collected anonymous, actual stories of emergent VR user and bystander interactions from both perspectives: the VR user (the person using the VR device) and the bystander (the non-VR person interacting with the VR user). The analysis of the stories revealed VR user

\footnotetext{
*e-mail: j.ohagan.1@research.gla.ac.uk

†e-mail:julie.williamson@glasgow.ac.uk

†e-mail:mark.mcgill@glasgow.ac.uk

§e-mail:mohamed.khamis@glasgow.ac.uk
}

and bystander interactions can currently be categorised into one of three categories: Coexisting, Demoing and Interrupting. Identifying impediments encountered during these interactions, we report empirical evidence concretely showing that VR users are sometimes surprised/scared when interrupted, that accidental collisions between the VR user and bystander do occur, and that bystanders often experience difficulty verbally communicating with VR users. We also highlight how partial (e.g. headphone removal) or complete (e.g. headset removal) transitions to reality by the VR user to interact with the bystander are not uncommon and we discuss what implications this might have for designers of systems to facilitate VR user and bystander interactions.

Our analysis also revealed that bystanders play an important role in moderating the VR user's experience, for example by intervening to save the VR user from potential harm by redirecting them away from nearby objects or people. But this position of power also creates the potential to exploit the vulnerable state of the VR user and we report several instances where the VR user's vulnerable state was exploited in abusive ways by a bystander - these ranged from recording an unaware VR user to physical assault by pushing the unaware VR user over. However, this capacity for abuse, whilst heavily skewed towards bystanders currently, is bidirectional. We reflect on the relationship between VR users and those observing or interacting with them from reality, and how this relationship might change in the future as VR headsets become more reality-aware [40].

\section{Related Work}

There has been three main lines of work in the area of studying interactions between VR users and bystanders: (1) work to increase a VR user's awareness of bystanders, (2) work to facilitate VR user and bystander interactions, (3) work to investigate VR user and bystander interactions in everyday environments outside the lab.

\subsection{VR user awareness of bystanders}

As VR overrides the visual, and often auditory, senses of the user, interactions with nearby people can be problematic $[14,34]$. To overcome this, research has seen the development of awareness mechanisms / cross-reality systems to facilitate interactions between VR users and bystanders. McGill et al. were the first to investigate how to automatically notify a VR user of nearby bystanders by contextually blending a photoreal view of bystanders into a VR scene [34]. Their approach, however, was found to significantly disrupt the VR user's sense of presence and they concluded less disruptive notifications were required. Since McGill et al's seminal paper, much work has investigated a range of approaches for increasing a VR user's awareness of bystanders. Research has investigated the feasibility of text notifications [40,45], audio notifications [40], haptic notifications [14], avatar designs [36, 48, 54], continuous vs one-off discrete notifications $[36,40]$, creating implicit auditory awareness [40], and contextually increasing the amounts of reality shown within the VR scene [13].

However, despite much work exploring how a VR user's awareness of bystanders can be achieved, the approaches taken thus far have largely focused on technology-facilitated awareness for the VR user. All were designed, built and evaluated in the context of the lab 
with minimal empirical evidence of the interaction being designed for being utilised in the design process. Some previous work studied perceptions of VR users during daily use. For example, McGill et al. surveyed usability challenges consumers faced while using VR headsets [34], and Ghosh et al. conducted user requirements elicitation into which elements of reality to prioritise increasing awareness of [14]. Absent, however, is empirical evidence of the interactions being designed for in-the-wild. As such there is a limited understanding of how awareness is currently facilitated, and particularly how this is achieved in non-technology mediated ways.

\subsection{Bidirectional awareness and interactions between VR users \& bystanders}

While investigating how to increase a VR user's awareness of bystanders is a popular topic of active research, work has also begun to investigate systems to facilitate interactions between the two. Yang et al's ShareSpace system considered how a VR user and bystander might coexist in the same physical space and investigated how to allow the bystander to section off areas of the space as their own [57]. Scavarelli et al. investigated notifications to prevent collisions between a VR user and nearby persons [46]. Others, meanwhile, have explored augmented reality (AR) systems to allow a bystander to better visualise the VR user's view in VR $[35,44,50]$. Gugenheimer et al. took this a step further and built novel, cross reality experiences to encourage a bystander to directly interact with a VR user's virtual environment $[15,18,19,22]$. Gugenheimer et al's FaceDisplay system presented a modified VR headset consisting of three, frontfacing, touch sensitive displays combined with a depth camera [19]. Nearby bystanders were able to view the VR user's virtual environment through the displays and could directly interact with it via touch or gestures. Mai et al. also investigated front facing displays on VR headsets, however, their approach focused on encouraging communication with the VR user by presenting the illusion of a transparent headset to bystanders $[25,26]$. They found their system had a positive effect on social presence and highlighted the need to consider how VR's introduction within the household might create social separation amongst its members. However, as with the work on increasing a VR user's awareness of bystanders, all of these systems to facilitate VR user and bystander interactions have been evaluated entirely within the lab. As such, without a full understanding of how VR user - bystander interactions occur in-the-wild it is hard to ascertain to what extent these interventions are even necessary and what types of interactions should be better supported and facilitated.

\subsection{VR in everyday environments}

While much work has investigated VR user and bystander interactions in the lab, little has been done to explore interactions outside of it. What work has been done has primarily focused on the social acceptability of VR in public spaces [7, 16, 24, 27] or on public transport $[2,41,47,55]$. Some have investigated bystander behaviour around VR users in these public spaces [16] or explored how bystanders might interrupt a passenger using VR on public transport [55] but missing from the discussion is how VR user and bystander interactions occur in more private settings such as home and workplace. As these are often the intended settings that systems to increase VR user's awareness of bystanders and facilitate interactions are framed to be used in, its absence from the discussion is surprising. As a first step toward investigating VR user and bystander interactions in these settings Dao et al. categorised "VR fail videos" posted online to understand why accidents occurred while using VR [6]. They identified several "failure types" (accidental collisions, falling over, excessive reactions), the causes of failure (fear, sensorimotor mismatch, spectator participation) and spectator reactions to the failure (laughter, concern, and support behaviours). These VR fails videos which comprised the data set used by Dao et al. are valuable in illustrating significant usability failures. However by their very nature videos that have been shared online for (at least in-part) entertainment purposes introduce a selection bias in the data set (noteworthy, shocking, or entertaining incidents) and consequently may portray a more exaggerated and skewed picture of the everyday challenges faced by VR users. Absent are the more grounded interactions which are not posted online as they offer little entertainment value for a potential viewer. What VR Fails confirms however is a need for more data on the prevalence, type, and magnitude of cross-reality challenges faced. We designed our survey with this in mind and eventually settled on an exploratory, story collection approach as our method of investigating VR user and bystander interactions in-the-wild.

\subsection{Summary}

As highlighted by the related work, there exists a large body work studying the interaction between VR users and bystanders. However, the vast majority of this work has been conducted entirely within the a laboratory setting. What work has explored VR user and bystander interactions outside the lab has failed to take into account are more mundane real experiences and usability challenges faced, focusing instead on (as Dao et al's work illustrates [6]) more extreme or obvious impediments. This motivated our use of real stories from VR users and bystanders to develop a broader picture of their interactions outside the lab, and obtain empirical evidence of how interactions between VR users and bystanders occur in-the-wild.

\section{SURVEY}

We collected a large number of diverse experiences from both VR users and bystanders to investigate interactions between the two inthe-wild. For this exploratory approach, we used an online survey.

\subsection{Design \& method}

We devised a survey to collect stories of actual VR user and bystander interactions based on the critical incident technique [9], which allows for "generating a comprehensive and detailed description of a content domain" [56] where participants are asked to recall and tell a story of an experience they have had related to an incident of interest being studied. We chose this approach as prior work has shown asking sensitive questions in self-report should be done in an indirect and anonymity-preserving way to minimise social desirability bias $[28,29]$. For example, we refrained from using words such as "abuse" or "spy" when referring to bystanders' possible actions towards the VR user. We also wanted to avoid any particular context and to minimise recall bias within our responses as much as possible. For example, if we had specifically highlighted using VR in home settings, participants may have been biased to recalling situations that happened in households. To address this, we refrained from specifying contexts throughout the questionnaire. The authors iterated through the questionnaire to ensure any questions that induce recall or social desirability bias are reworded.

The questionnaire was designed in an iterative process and pilot tested using small participant samples $(\mathrm{N}=15)$. The critical incident was presented as a sketch of an intentionally generic VR user and bystander interaction with two stick-figures labelled VR user and non-VR bystander, shown in Figure 2, alongside a short description which gave clear, labelled, anonymous roles for participants to use in the stories if desired. We did not specify any particular "type of interaction" between the VR user and bystander to avoid giving participants the impression there was a "correct" type of interaction we wanted them to describe and so potentially discourage them from describing others.

\subsection{Questionnaire structure}

An overview of the survey structure is provided in Figure 1. The questionnaire first determined whether participants had a story to 


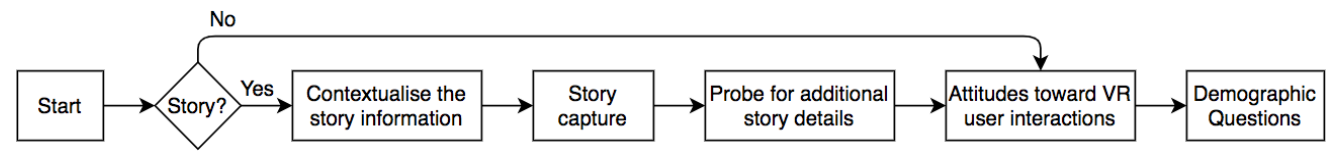

Figure 1: Flowchart outlining the survey structure

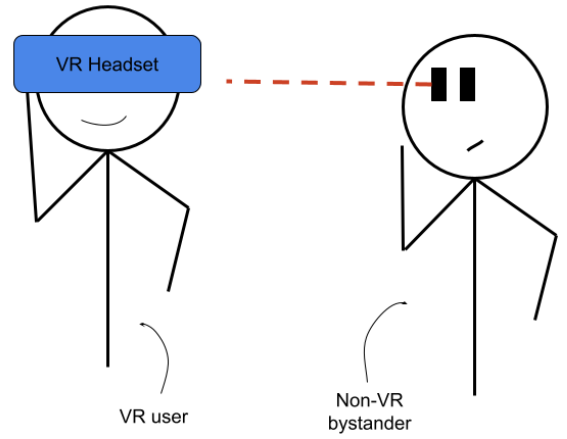

Figure 2: The sketch used in the survey. Presented alongside the following description: "With the rise in popularity of VR headsets, interactions between VR users and non-VR bystanders are becoming more frequent. However, little is currently known about how VR users and nearby people interact with one another. The goal of this survey is to capture stories of real experiences you have had as a VR user or bystander when interacting with the other."

share. The sketch and description (Figure 2) were displayed and participants were asked to state whether they had experienced or observed a real situation similar to this (Yes: as the VR user, as the non-VR bystander, as both VR user \& non-VR bystander, as a third party observer or No). If "No", participants were automatically routed to the final part of our survey where we investigated participants' attitudes towards interactions with a VR user as a bystander using a set of Likert scale questions. Note: no participant in the data set analysed in this paper selected this option.

If "Yes", participants were then asked a series of questions to contextualise their story: how often they experience a situation like this, how frustrated they typically are during these interactions, and how often they feel the need to interact with a VR user. We then asked participants to describe the situation in as much details as possible via free text entry. Next we enquired about specific details which would allow us to indirectly derive further insights about the situation: where the interaction took place, what the VR user / bystander did, how they reacted, if they knew each other and who the participant was (the VR user, bystander or a third party). We used multiple choice where appropriate, but most of the questions allowed for free text entry. Participants were then asked 5-point Likert scale questions to investigate participants' attitudes towards interactions with a VR user as a bystander. Note, due to space constraints, we do not report the results of this section within this paper. As this section occurred after the questions analysed in this paper they have no influence on how participants answered the analysed questions.

Finally, participants completed standard demographic data questions and were asked to indicate their prior experience with VR headsets using a 5-point Likert scale. At the end of our survey we provided participants with the option to share additional stories through a link to a second survey. This second survey only included the questions from our survey relevant to contextualise, capture and probe participant's story and questions to capture participant's demographic data. No participant opted to share an additional story. Participants could also optionally sign up for a raffle for two online shop vouchers. The study was approved by our ethics committee.

\subsection{Limitations}

Self-report is a common and established approach in a diverse range of research areas [1,23], however, it is not without its limitations. Self-reported data is susceptible to inaccurate statements, influence by wording or recall bias [42]. Additionally, when asking people to self-report potentially sensitive experiences, social desirability can introduce error [51]. Also, a single study cannot claim to include all possible interactions between VR users and bystanders.

Although we cannot rule out any of these limitations entirely, we have taken measures to minimise their potential impact. (1) Our questioning approach allowed for anonymity and questions were designed in an iterative process. (2) To identify invalid responses, we manually inspected all collected responses before analysis. (3) We captured a broad range of feedback for greater ecological validity when contrasted with other published datasets, e.g. "VR Fails" [6].

\section{AnALYSIS}

We analysed our data to investigate the types of interactions reported as occurring between VR users and bystanders within our data set and to determine the emergent themes within it. Respondent answers were coded using initial coding [4] where respondents' statements were assigned emergent codes over repeated cycles with the codes grouped using a thematic approach. Multiple coding was allowed meaning statements could be encoded as multiple categories, e.g. [for location data] P48: "Exhibitions, home, work" was coded as "Public Spaces", "Home" and "Office / Workplace". A single coder performed the coding and reviewed the coding with two other researchers to resolve unclear codes and discuss the depth and specificity of codes. Two coding cycles were completed.

Prior to the analysis we inspected the data to remove invalid responses from the data set. Responses which were entirely blank or lacked sufficient detail were removed (e.g. an initial story of a few words and then blank responses to all follow up questions). We also checked whether participants responses were coherent throughout (e.g. the responses to the follow up questions were consistent with the initial story described). This review process flagged 16 responses which were removed from the data set. This left us with the 100 valid stories reported on in this paper.

\section{REsULts}

\subsection{Participant demographic data}

We distributed the survey through mailing lists and social media. To ensure ecological validity, we advertised the questionnaire on a variety of different platforms including subreddits, Facebook groups, VR discord groups, and XR mailing lists. Participants who gave a valid story were offered the option to enter a raffle for two online shop vouchers. 100 respondents (31 female, 68 male, 1 fluid) aged between 16 and $68(\mathrm{M}=27.96, \mathrm{SD}=8.13)$ completed the survey. $\mathrm{Re}-$ spondents were asked to indicate their prior experience with VR headsets using a 5-point Likert scale ( $1=$ none; $5=a$ lot $),(M=3.91$, $\mathrm{SD}=1.05)$. All participants indicated they had at least "a little (2)" experience with VR.

\subsection{Contextualising our story data}

\subsubsection{Experience with VR user and bystander interactions}

Most participants (73 of 100) indicated they had experienced or observed an interaction between a bystander and VR user in situations where they were the bystander and in other situations where 
they were the VR user (question 1, table 1). When asked how often they had experienced these interactions using a 5-point Likert scale $(1=$ never; $5=a$ lot $)$, most participants indicated it was something which "occasionally" happened to them $(\mathrm{M}=3.21, \mathrm{SD}=0.99$, question 2 , table 1). We also asked how often they felt the need to interact with a VR user when around one (1=never; $5=$ all the time) and again most indicated this was something which "occasionally" happened ( $\mathrm{M}=2.61, \mathrm{SD}=1.04$, question 3, table 1). Finally, we asked participants how frustrated they felt during interactions with VR users ( $1=$ not at all frustrated; $5=$ very frustrated). Surprisingly, as shown in question 4 , table 1 , most participants indicated these interactions were not typically frustrating $(\mathrm{M}=1.63, \mathrm{SD}=0.85)$ with 88 of 100 indicating they were at most "slightly frustrated" during interactions with VR users. This was unexpected, as "problematic interactions" are often used to justify building systems to facilitate interactions between the two $[25,34]$ and it suggests that in-the-wild evaluations of such systems may become increasingly important to obtain a more accurate picture of where frustrations actually exist and are mitigated against.

Finding 1: Interactions between bystanders and VR users are not as frustrating as often as implied in prior work.

\subsubsection{Contextual Metadata}

To better contextualise our story data, we asked participants 3 questions to provide a general overview of the interaction being described. First, we asked if the story the participant described involved physical contact and/or verbal communication, question 5, table 1 . Verbal or verbal communication combined with physical contact accounted for most interactions. Next, we asked if the VR user and bystander knew each other, question 6 , table 1 - most did. Finally, we asked participants to state their role within the story they reported, question 7, table 1. This showed we had an almost even split of VR users and non-VR bystanders.

Finding 2: Most interactions involve VR users and bystanders that know each other. Verbal and the combination of verbal communication and physical contact seem to be the most commonly used forms.

\subsubsection{Interaction Location Metadata}

We also asked participants for the location of the interaction. From this we identified 4 high level location types (Table 2). In total 111 locations were mentioned by participants with 94 participants stating one location and 6 stating multiple locations. As expected, most occurred within the home although 29 instances of locations outside the home were reported by participants. 20 of these were work settings (Office / Workplace and Universities / Research Labs) while 9 were public spaces (composed of Conference-type events: 4 , VR arcades: 3, Museums: 1, Planes: 1).

\subsubsection{Interaction Types Metadata}

We first analysed our data by categorising the general types of interactions described by participants to give a high level overview of the types of interactions captured in our data. We identified 3 high level interaction types:

- Coexisting: the bystander and VR user share the same physical space and one interacts with the other for some reason

- Demoing: the bystander is demonstrating VR to the VR user

- Interrupting: the bystander interrupts the VR user
(1) Have you experienced or observed a real situation similar to this?

Count

\begin{tabular}{ll}
\hline Yes, I was the VR user: & 23 \\
Yes, I was the non-VR bystander: & 4 \\
Yes, as both the VR user and non-VR bystander: & 73 \\
Yes, I was neither but I observed such a situation: & 0 \\
No: & 0 \\
\hline (2) How often have you experienced & Count \\
a real situation like this? & 0 \\
\hline Never: & 27 \\
A little: & 38 \\
Occasionally: & 22 \\
Often: & 13 \\
All the time: & \\
\hline
\end{tabular}

(3) How often have you felt the need

to interact with a VR user?

Count

Never:

15

A little: 15

Occasionally:

Often:

34

All the time:

15

4

(4) How frustrated do you feel during VR user and non-VR bystander interactions?

Count

Not at all:

Slightly frustrated:

Somewhat frustrated:

Frustrated:

Very frustrated:

54

54
34

9

2

(5) Did your story involve verbal communication and/or physical contact?

Count

Physical contact and verbal communication: 49

Verbal communication only: $\quad 46$

Physical contact only:

It involved neither:

5

0

(6) Did the VR user and non-VR bystander

know each other?

$\begin{array}{ll}\text { Yes: } & 93 \\ \text { No: } & 6 \\ \text { Not sure: } & 1\end{array}$

(7) Were you the VR user, the non-VR

bystander or someone else?

I was the VR user:

Count

I was the non-VR bystander:

51

I was neither (a third party):

49

Table 1: The collection of questions to contextualise participants stories and the participants' responses.

\begin{tabular}{ll}
\hline Where did the story take place? & Count \\
\hline Home: & 82 \\
Office / Workplace: & 11 \\
Universities / Research Labs: & 9 \\
Public Spaces: & 9 \\
\hline
\end{tabular}

Table 2: The coded locations of where the interactions described occurred. As expected, most were at home although a variety of locations outside the home were also mentioned. Some participants provided more than one location. 
While most stories contained only one high level interaction type we did allow stories to contain multiple as shown in part 1 of table 3, e.g. P30: "New or casual users needing assistance with controls, game direction, or just chatting. When I'm playing, my girlfriend and I will chat" was coded as "Coexisting + Demoing".

Coexisting: was our most prevalent interaction type - included within 54 stories, with 79 scenarios described, part 2 of table 3 . We identified a range of interactions within this theme, all of which highlight the diverse range of ways VR is already being used around other individuals: (1) as something to ignore, (2) as something to observe, (3) as something to directly engage with.

Demoing: was included in 36 stories indicating that despite VR is being used more regularly within the home that showcasing the technology to individuals for the first time remains a regular interaction for some.

Interrupting: was included in 21 stories with part 3 of table 3 breaking down how interruptions occurred. The preference for verbal interruptions matches results from prior work on VR user bystander interruptions conducted in the lab $[10,41]$ although the combined use of speech and touch was theorised [41] to occur more frequently than was reported. Participants justifications for the combined use of speech and touch when interrupting matched those given by participants who used a similar combined approach in the lab [41], that is, to convey location alongside existence when interrupting, P41: "Verbal and physical communication was used to establish presence and location".

Finding 3: Most VR user-bystander interactions can currently be classified to coexisting, demoing, and interrupting.

\subsection{Bystander interactions with VR user}

\subsubsection{Interruptions}

Only 8 of our interruption stories explicitly stated the VR user was surprised by the bystander's interruption. 5 of these involved touch, 3 involved speech alone. The latter surprised us as speech alone is not typically thought of as a means of surprising or scaring a VR user when interrupting. Instead, touch is viewed as the mostly likely method of eliciting this reaction $[10,41]$.

Finding 4: VR users are sometimes (but not always) surprised by the bystander's interruption. Most surprises are when the bystander uses physical contact, but there are also cases where the user is surprised by verbal communication.

Interestingly, 1 story described the bystander as waiting for the optimal moment to interrupt the VR user, P31: "She [the bystander] knows she have small windows of opportunity to reach me, usually between songs in Beat Saber... Waits for opportunity to reach me based on the amount of waving of my arms". This story is similar to the work of George et al. who investigated whether bystanders could identify low activity moments in a VR user's application usage which they posited were the ideal moments for interruption [10]. While prior work assumed this behaviour takes place in a lab study [10], our finding confirms that it indeed takes place in real world scenarios.

Finding 5: Bystanders sometimes use cues, such as audio from the headset or the VR user's movements, to identify the optimal interruption opportunity.

\subsubsection{Problems guiding VR users}

37 stories reported scenarios where the bystander was demonstrating VR to the VR user. 24 involved the bystander assisting the VR user

\begin{tabular}{ll}
\hline (1) High level story classification & Count \\
\hline Coexisting: & 43 \\
Demoing: & 28 \\
Interrupting: & 17 \\
Coexisting + Demoing: & 8 \\
Coexisting + Interrupting: & 3 \\
Demoing + Interrupting: & 1 \\
\hline (2) How coexisting occurred & Count \\
\hline Coexisting: & $\mathbf{7 9}$ \\
- Bystander ignores VR user / converses with them & 21 \\
about things unrelated to VR experience: & \\
- Bystander watches / reacts to VR user: & 43 \\
- Bystander and VR user take turns using headset: & 10 \\
- Bystander plays multiplayer game with VR user: & 5 \\
\hline (3) How interruptions occurred & Count \\
\hline Interrupting: & $\mathbf{2 1}$ \\
- Verbal communication alone: & 12 \\
- Physical contact alone: & 5 \\
- Verbal communication and physical contact: & 4 \\
\hline
\end{tabular}

Table 3: The coded classifications of the interaction type metadata. (1) shows the story classification for the high level types of interactions that occurred. Each story maps to 1 code, e.g. Coexisting includes stories containing only the Coexisting theme whereas Coexisting + Demoing contains stories with instances of both themes (themes explained in section 5.2.4). (2) breaks down our Coexisting theme: within the 54 stories in this theme 79 scenarios were described. (3) Looking at how bystanders interrupted VR users, our data shows most used verbal communication.

with the controls of the application. Issues with controlling VR stemmed from unfamiliarity with the controller / being unable to see it while in VR, P5: "When using the headset, controls arent visible", and because individuals misunderstood how the controls worked, Participant 94: "a lot of people new to VR seem to think it's just shake controls like on the original Nintendo Wii". To solve these issues, the bystander was required to intervene and show the VR user how to use the controller, P30: "they usually ask for help with control and $i$ will place their hands in the proper position".

21 stories had the bystander providing verbal instructions to guide the VR user through the experience, P1: "I was sat next to them giving them instructions of what to do in the VR game". Frustration was created here due to the VR user being unable to easily point at objects in the VR user's virtual environment (6 stories), P33: "trying to explain/point out/help them can be frustrating when they can't see what you're indicating or they aren't looking at what you need them to". As a consequence of this, the process often becomes trial and error-like, P9: "trying to indicate an action like look up, no there, to the left, not so low, up...”.

\subsubsection{Auditory occlusion of bystander activity}

12 stories reported a VR user having difficulty hearing a bystander's attempt to verbally interact with them. 8 of these described situations where the bystander was required to shout as the VR user could not hear them over the VR application's audio, P27: “The bystander usually tries to help them by yelling at them (often trying to be louder than the game in the VR users ears)". The remaining 4 indicated the VR user missed the bystander's verbal interaction entirely, P17: "They'll call to me, which under normal circumstances would get my attention, but whilst in VR, I often can't hear them". Both of these provide real world evidence which reinforces work by Ghosh et al. who identified awareness of nearby audio as one of the key elements of reality VR users wished to be informed of [14], and further motivates the creation of implicit auditory awareness as a direction of future work [40]. 


\subsubsection{Pets are problematic too (non-human bystanders)}

Although we asked participants about interactions with bystanders, 2 participants discussed interactions with pets in addition to bystanders, P41: "Pets don't really understand VR, and its not uncommon for a cat or dog to come in and sit by my feet while I'm using a VR headset. This can cause some minor issues, like tripping or prodding".

Unlike humans, pets are unaware of the human's lack of visibility of reality, and consequently may put themselves or the VR user in danger. Prior work which has explored increasing a VR user's awareness of their surroundings has focused on nearby objects and people. Absent, however, are pets despite how common they are within the household [43]. Furthermore, work is beginning to explore increasingly complex technology for interactions with animals [20] and future work can also consider how novel experiences and safe usage between XR users and animals could be achieved.

\subsection{Bystander's position of power over VR users}

\subsubsection{Bystander recording VR user}

2 participants said they had recorded video of the VR user. Both indicated their intention was not malicious, rather, it was a natural response because of how enjoyable watching VR user's reactions was, P73: "I saw many phones recording the experience [watching the VR user]... I didn't know it would be so much fun watching just reactions from vr users from my friends and family". As highlighted by Dao et al's work [6] the recording of VR users is not an uncommon phenomenon. Missing, however, is the VR user's perspective - are they comfortable with their actions being recorded without their consent or knowledge and potentially shared online? Furthermore, although our participants indicated their intentions were not malicious this is not always true. Bystanders may shoulder surf VR authentication [11,12,31,32] and record the VR user for their own malicious usage, e.g. attempting to shame or embarrass the VR user by posting the video online. This reinforces the need to develop of usable, secure authentication systems for VR which are resistant to attacks where the bystander records the VR user's actions.

\subsubsection{Abusing their position of power}

Prior work [41] has reported situations where a bystander will take advantage of a VR user's vulnerable state and improvise a novel, unconventional interaction with them. 12 such interactions were reported as occurring within our participant stories. 1 story mentioned that the bystander mimicked the VR user's actions which they thought were amusing, P19: "The bystander is staring at me at a distance, laughing and mocking my physical movements". 10 stories described scenarios where the bystander touched the VR user to intentionally scare or tease them, P47: "sometimes the friend outside of VR would want to tease or scare the friend in $V R$ by touching them". 9 of these were spur of the moment decisions upon seeing the vulnerable state of the unaware VR user. 1, however, was in direct response to the VR user's experience which the bystander had observed, P49: "in a scenario on top of a mountain, I moved towards the edge of a cliff to see how realistic it was. Just as I was nearing the precipice, one of my friends pushed me 'off the cliff' to my doom, and then laughed at my misfortune. This was very frustrating". Finally, 1 story saw the bystander playfully surprise the VR user in an attempt to contribute to their experience in VR. Here the VR user, whose application took place in a candy-like land, was surprised by the bystander who fed them a cookie to eat, P83: "I got a cookie. Them walked over to him and told him to open his mouth. Without telling him what it was I put the cookie in his mouth.".

While the last story is an example of a positive interaction between the bystander and VR user, the aforementioned 11 are more exploitative in nature. While some stated their intention was to "tease" the VR user in a likely playful manner, all are reliant on exploiting the VR user who is in a compromised state where their awareness of their surrounding area is impaired. In this state the VR user is more vulnerable to acts by others and a power imbalance is created between the VR user and bystander which can be exploited. In our stories, the more malicious interactions described ranged from "teasing" to abusive physical contact (e.g. pushing an unaware VR user) but other more exploitative behaviour such as theft of personal possessions or more abusive physical assault could have occurred. These are typically not the types of interactions highlighted as occurring between bystanders and VR users, however, as our results highlight they do occur and should not be treated lightly.

Finding 6: Bystanders to occlusive VR experiences hold a position of power over the VR user - both beneficial (e.g. for safety) but also open to abuse.

\subsection{VR user interactions with bystanders}

\subsubsection{Physical transitions to reality}

19 stories indicated the VR user transitioned (either fully or partially) to reality to interact with the bystander. 6 were partial transitions: the removal of headphones ( 2 stories) and temporarily peeking out form under the headset (4 stories), P42: "Pull the speaker away from one ear to better hear them because it's usually a little too loud". The remaining 13 were full transitions to reality: turning on the passthrough view of the headset ( 2 stories) and complete removal of the headset (11 stories), P6: "I [the VR user] reacted by removing my headset, and going off to talk to them". It is not known what motivates such behaviour and how it might impact work seeking to increase the VR user's awareness of their surrounding area by gradually increasing the amount of reality incorporated within the VR scene $[13,34]$. Further work in understanding the user's motivation will clarify whether such systems are warranted.

Finding 7: Some VR users will transition (either partially or fully) to reality to interact with the bystander.

\subsubsection{Bystander becomes a haptic proxy}

1 story highlighted how the VR user and bystander can collaborate to intentionally create a novel experience for the VR user, $P 84$ : "I don't remember how [we] thought of it but we ended having sex with him wearing the headset. The Waifu game let you set the Avatar anywhere in the play space and had animations so that it would move. So he laid down and I got top and he set the Avatar in the right place.". This shows the effort some will go to create novel experiences for each other and its use of the bystander as a haptic proxy is similar to Cheng et al's Haptic Turk system where 4 individuals manually carried, tilted and pushed a player's limbs / torso to provide haptic feedback for their gameplay experience [3].

Although the interaction described by our participant, and the prior work, were consensual experiences, a more cynical view of this story can also be taken. While the participants in our story were exploring potential novel interactions others might take this use of substitutional reality [49] to unhealthy extremes and replace how they perceive the entire world around them. This also says nothing for the potential exploitation of the proxy person who is reduced to a placeholder for the fantasies the VR user wants to / is able to reenact. Additionally, the line between consensual fun and abuse is not entirely clear here. What if the proxy person wishes to revoke consent but the VR user misses their attempt to do so (e.g., due to the headset causing auditory or visual occlusions) and the proxy person is not in a position to physically stop the VR user. What if this interaction facilitates unhealthy sexual relationships where the 
proxy person is exploited for the pleasure of the VR user. While it is "all fun and games" now it is not difficult to imagine scenarios where this is exploited and abused.

Finding 8: Bystanders may collaborate with VR users to create novel experiences by, for example, providing haptic feedback. This also has the potential to be exploited by either party to abuse the other.

\subsection{Managing the physical space}

\subsubsection{Intervention by others}

In 28 stories, participants said they felt it was the bystander's role to manage the physical space of the VR user. 19 of these reported situations where the bystander directly managed the physical space of the VR user. This involved the bystander watching the VR user and any other nearby bystanders and either: (1) redirecting the VR user back into the play area away from objects / people in the nearby area, P46: "I'll let my friend know if they need to recenter themselves, or if they're too close to a chair or dresser", or (2) redirecting nearby bystanders away from the VR user's play area, P21: "[the VR user] was playing Superhot on the headset, while the others [the bystanders] were watching. However, the others were in the way, so I had to move them while my friend played while also avoiding her myself",

The remaining 9 discussed the need for bystanders to be mindful of the VR user - that it was the bystander's responsibility to maintain a safe distance from the VR user and negotiate when entering the VR user's play area, P37: “There are some courtesies I think people should adopt when interacting with a VR user, and that's generally not standing close enough to get accidentally hit, or unwanted or unexpected touching". Exploring these expected courtesies of VR user and bystander interactions in more detail is an opportunity for future work and is a natural progression of the existing work done into how bystanders interrupt VR users $[10,41]$.

Finding 9: Bystanders perceive the safety of the VR user as their responsibility and may intervene to save the VR user from potentially dangerous situations.

\subsubsection{Collisions with reality}

Despite some bystanders acting as an overseer of the physical space accidental collisions with nearby objects or people do occur [6] and require built-in safety systems to try and prevent them $[38,52]$. However, only 5 such unintentional collisions with reality were reported in our data set, all of which feature accidental contact between the bystander and VR user, P60: "While my girlfriend was watching me play a VR game, she came too close to my play area, resulting in me accidentally striking her with my controller". All 5 followed this pattern, where the bystander approaches or attempts to navigate past the VR user and is accidentally struck by them. This was somewhat surprising as Dao et al. reported many of their " $V R$ fail" videos were such accidental collisions with reality [6] while our stories indicate it is much more frequent for bystanders to intervene and prevent such collisions rather than allow them to occur (albeit off camera).

\subsubsection{Physical indicators of the VR user's playspace}

Interestingly, 2 participants described going as far as including visible indicators of the VR user's play area within their real world environment. Both indicated they used a rug / carpet to act as a visible identifier for bystanders of the VR user's play area, P23: "She [the bystander] knows not to step on the carpet as I may accidentally make physical contact unintentionally". This highlights some of the creative solutions individuals develop to accommodate the introduction of VR in their home. Similar creative solutions have been seen with interruptions where individuals develop custom interruption systems to meet the needs of their particular household [41]. At present, it remains unknown to what extent the introduction of VR in the home and workplace will create or widen accessibility problems between individuals in the these settings. Investigating these and developing solution to rectify the problems is another direction future work should consider.

Finding 10: VR users and bystanders sometimes negotiate physical boundaries using physical items around them such as rugs.

\section{Discussion \& FUtURE WORK}

We close by discussing results from our analysis and by highlighting directions future work can explore.

\subsection{Pertinent challenges for future work}

Although our participants indicated they did not consider VR user bystander interactions particularly frustrating, we identified several problematic scenarios within the interactions that future work can consider. Multiple stories reported situations where the VR user was surprised/scared due to bystander interruption. Although this itself is not surprising it does provide empirical evidence to further motivate the development of systems to automatically notify the VR user of nearby bystanders $[14,34,40]$. By preemptively warning the VR user someone is nearby the risk of being surprised by the bystander is reduced.

Noteworthy also was the preference for verbal communication to be used while interrupting. This, paired with the difficulties experienced by some VR users to hear a nearby bystander who is speaking to them, motivate the need to consider how auditory awareness can be increased. Despite Ghosh et al. also highlighting the desire for VR users to have an increased auditory awareness of their surroundings [14] little work has considered how this could be achieved [40]. Instead the majority of systems developed thus far have focused on increasing the VR user's visual awareness of bystanders [34,54] and exploring how alternative forms of awareness, such as auditory awareness, can be achieved is a direction future work can explore.

A second problem experienced during verbal interactions between VR users and bystanders was the difficulty bystanders faced in giving verbal instructions and directions to the VR user. Because bystanders lack a means of pointing or highlighting content within the VR user's virtual environment the task of giving instructions to the VR user often reduces to a trial-and-error process. However, systems to facilitate such interactions have been already explored for specialised applications in industry. For example, Ibayashi et al's Dollhouse VR proposed a tool for architects to allow a VR user to see a first-person view of a home while non-VR bystanders could modify its design in real-time [21]. To facilitate communication between the VR user and bystanders Ibayashi et al. developed a range of interaction techniques such as object highlighting and pointing tools. Exploring how similar tools could be developed to facilitate VR user and bystander interactions within the home is another direction future work can consider.

Another problem experienced by many of our participants was the difficulty experienced by first time VR users in learning the controls of the VR system. While some were due to individuals misunderstanding the controls conceptually (e.g. mistaking it for a Wii-like motion controller) others were simply due to the individual not knowing the layout of the controller and being unable to see it while in VR. As such the individual resorted to either peeking out from under the headset at the controller or requesting the bystander 
manually position their fingers on the correct button. Future work can also investigate how VR controls can be made more accessible for those unfamiliar with the controller layout. Is simply allowing the VR user to see an in-VR controller model sufficient? Can systems be built to contextually detect and turn on this view on-the-fly? Improving the interaction between a VR user and bystander does not always require building systems to facilitate the interaction. If solutions can be built to prevent problems from occurring (e.g. asking for controller assistance) then these should be explored also.

\subsection{Why do VR users take off their headsets to commu- nicate with bystanders?}

In response to a bystander initiating an interaction with the VR user in 19 of our stories the VR user transitioned to reality to interact with the bystander. For some, this was a partial transition (e.g. removing the headphones, peeking out from under the headset) whereas for others it was a full transition (e.g. turning on the passthrough view, removing the headset entirely). It is unknown what motivates this behaviour, particularly those who desired a complete transition to reality and favoured headset removal instead of utilising the passthrough view feature of VR headsets. It may be because their respective headset lacks such a feature or their unawareness of it. It may also be because the user is not motivated to turn on the passthrough feature, because it is faster to remove the headset, because the VR user finds it cumbersome or socially awkward to wear it during interactions or because they desire bidirectional eye contact during their interaction with the bystander. While Mai et al. have investigated how an artificial face imposed over the headset might encourage bystanders to interact with the VR user $[25,26]$ they have not considered whether such systems allow the VR user to fully express themselves during interactions. Future work might also investigate what shortcomings users have with the existing passthrough view systems (e.g. the Oculus passthrough view [38] and the Window's mixed reality flashlight [37]). Investigating their usage and comparing them directly may identify user experience problems in them and highlight further opportunities for future work.

\subsection{The (current) role of bystanders - great power, and significant responsibility over VR users}

An overarching connection throughout several of our themes is that the bystander has complete awareness (and to some degree control) over the VR user and that this control is used both positively and negatively. Positively, bystanders will perceive the VR user's safety as their own responsibility and intervene to save the VR user from potentially dangerous situations. However, some will do nothing instead, allowing the VR user to harm themselves [6] or worse abuse this position of power and harm the VR user. Throughout our results we identified a range of scenarios where this power imbalance can be exploited by a malicious bystander.

For example, 2 of our stories reported the unsolicited recording of the VR user by bystanders. While some instances of bystander recording a VR user will be consensual, our stories highlight how bystanders have the capacity to capture and share video of an unaware VR user without the VR user's consent. Bystanders might also simply observe the VR user's activity without the VR user's knowledge they are copresent (a story type not likely captured by our survey due to the one-sided nature of the interaction). While systems to increase a VR user's awareness of bystanders can assist with the latter $[14,34,36,40]$ these systems typically do not inform the VR user of the bystander's actions. Future work can consider exploring systems to notify the VR user in response to a specific bystander action then, e.g. when they take out their phones and appear to be recording with them $[34,39]$. This might draw from work in social signal processing [53] to build "smarter" notification systems to allow the VR user to specify how situational awareness of their surrounding area is increased. For example, the VR user might specify to only be notified of bystander existence should the bystander perform a specific action of interest, e.g. begins to record the VR user with their phone.

A second example highlighted in our results was the more direct abuse of this power position where actions ranged from teasing the VR user to physical assault by pushing an unaware VR user. Here the exploitation of the VR user's vulnerable state was more malicious in nature. While actions such as teasing are typically done in a playful manner and although 1 story showed how the power imbalance could be exploited in a positive way (surprising the VR user by feeding them a cookie), it is also not hard to imagine how this exploitative behaviour might escalate. For example, when in VR the user is at increased risk of their personal possessions being stolen $[17,30]$. Alternatively, a bystander might place furniture in the path of the VR user to force an accidental collision with the nearby object. They might even record the collision in an attempt to create a viral video [6]. Again this highlights the necessity of research into systems which increase a VR user's awareness of their surroundings, and the presence and activities of others (be they strangers or known to the user) $[14,34,36,40]$. While some of these potentially abusive scenarios may have been mitigated against by the availability of such systems, we suggest those outside VR also need to be educated to understand what is and is not appropriate with respect to consensual VR user interactions, with the current lack of established social norms perhaps enabling encounters that are of high risk to both VR users and bystanders.

Finally, as highlighted by our bystander becomes a haptic proxy story (Finding 8), it is not always the VR user who is the exploited individual within the interaction. Instead, situations can arise where the VR user is in a position to exploit the co-located person (the proxy person). Our results highlighted sexual activities as one such scenario. Here mutual trust is required between the VR user and proxy person, however, it is unclear where the line between fun and abusive, healthy and unhealthy is drawn. What if the proxy person is reduced to a placeholder for the VR user's fantasies. What if this facilitates unhealthy sexual relationships where the proxy person is reduced to a means of pleasure of the VR user. While it is consensual, novel, fun experiences for those involved currently, one can easily envision scenarios where this use of substitutional reality $[5,8,49]$ is taken to unhealthy and exploitative extremes.

Fundamentally, exploitation and abuse is possible from both parties, however the stories captured by our survey suggest, at present, bystanders currently have more power, and assume more responsibility, over the VR user. Despite this, our work is a snapshot of current behaviour around predominantly occlusive VR headsets, and we expect this power balance will shift over time, with new behaviours emerging as passthrough-enabled cross-reality interactions reach consumers, and bystanders become equipped with AR headsets potentially facilitating cross-reality bidirectional awareness between both parties. Further insights need to be captured as these changes occur, and this paper demonstrates a story driven methodology can reveal novel insights into user behaviour in this context.

\section{Conclusion}

Empirically describing the use of VR outside the lab remains an ongoing challenge for researchers. Through an exploratory story survey we investigated VR user and bystander interactions outside the lab in everyday environments. Our analysis reveals current VR user and bystander interactions can be categorised into one of three categories: Coexisting, Demoing, Interrupting. We identify common impediments encountered during these interactions and we highlight the important role bystanders play in moderating the VR user's experience by, for example, intervening to save the VR user from potential harm. However, this position of power also creates the opportunity to exploit the vulnerable state of the VR user and we report several instances of this occurring. 


\section{REFERENCES}

[1] A. Adams and M. A. Sasse. Users are not the enemy. Communications of the ACM, 42(12):40-46, 1999.

[2] L. Bajorunaite, S. Brewster, and J. Williamson. Virtual reality in transit: how acceptable is vr use on public transport? In 2021 IEEE Conference on Virtual Reality and 3D User Interfaces (VR), pp. 432-433, 2021.

[3] L.-P. Cheng, P. Lühne, P. Lopes, C. Sterz, and P. Baudisch. Haptic turk: A motion platform based on people. In Proceedings of the SIGCHI Conference on Human Factors in Computing Systems, CHI '14, p. 3463-3472. Association for Computing Machinery, New York, NY, USA, 2014. doi: 10.1145/2556288.2557101

[4] S. A. L. Corbin J. M. Basics of qualitative research: techniques and procedures for developing grounded theory. SAGE Publications, Inc., 1998.

[5] F. Daiber, D. Degraen, A. Zenner, T. Döring, F. Steinicke, O. J. Ariza Nunez, and A. L. Simeone. Everyday Proxy Objects for Virtual Reality. Association for Computing Machinery, New York, NY, USA, 2021.

[6] E. Dao, A. Muresan, K. Hornbæk, and J. Knibbe. Bad breakdowns, useful seams, and face slapping: Analysis of vr fails on youtube. In Proceedings of the 2021 CHI Conference on Human Factors in Computing Systems, CHI '21. Association for Computing Machinery, New York, NY, USA, 2021. doi: 10.1145/3411764.3445435

[7] P. Eghbali, K. Väänänen, and T. Jokela. Social acceptability of virtual reality in public spaces: Experiential factors and design recommendations. In Proceedings of the 18th International Conference on Mobile and Ubiquitous Multimedia, MUM '19. Association for Computing Machinery, New York, NY, USA, 2019. doi: 10.1145/3365610.3365647

[8] D. Englmeier, J. O'Hagan, M. Zhang, F. Alt, A. Butz, T. Höllerer, and J. Williamson. Tangiblesphere - interaction techniques for physical and virtual spherical displays. In Proceedings of the 11th Nordic Conference on Human-Computer Interaction: Shaping Experiences, Shaping Society, NordiCHI '20. Association for Computing Machinery, New York, NY, USA, 2020. doi: 10.1145/3419249.3420101

[9] J. C. Flanagan. The critical incident technique. Psychological bulletin, 51(4):327, 1954.

[10] C. George, P. Janssen, D. Heuss, and F. Alt. Should i interrupt or not?: Understanding interruptions in head-mounted display settings. In Proceedings of the 2019 on Designing Interactive Systems Conference, DIS '19, pp. 497-510. ACM, New York, NY, USA, 2019. doi: 10. $1145 / 3322276.3322363$

[11] C. George, M. Khamis, D. Buschek, and H. Hussmann. Investigating the third dimension for authentication in immersive virtual reality and in the real world. In 2019 IEEE Conference on Virtual Reality and 3D User Interfaces (VR), pp. 277-285, 2019. doi: 10.1109/VR.2019. 8797862

[12] C. George, M. Khamis, E. von Zezschwitz, M. Burger, H. Schmidt, F. Alt, and H. Hussmann. Seamless and secure vr.

[13] C. George, A. N. Tien, and H. Hussmann. Seamless, bi-directional transitions along the reality-virtuality continuum: A conceptualization and prototype exploration. In 2020 IEEE International Symposium on Mixed and Augmented Reality (ISMAR), pp. 412-424, 2020. doi: 10. 1109/ISMAR50242.2020.00067

[14] S. Ghosh, L. Winston, N. Panchal, P. Kimura-Thollander, J. Hotnog, D. Cheong, G. Reyes, and G. D. Abowd. Notifivr: Exploring interruptions and notifications in virtual reality. IEEE Transactions on Visualization and Computer Graphics, 24(4):1447-1456, April 2018. doi: 10.1109/TVCG.2018.2793698

[15] J. Gugenheimer, D. Dobbelstein, C. Winkler, G. Haas, and E. Rukzio. Facetouch: Enabling touch interaction in display fixed uis for mobile virtual reality. In Proceedings of the 29th Annual Symposium on User Interface Software and Technology, UIST '16, p. 49-60. Association for Computing Machinery, New York, NY, USA, 2016. doi: 10.1145/ 2984511.2984576

[16] J. Gugenheimer, C. Mai, M. McGill, J. Williamson, F. Steinicke, and K. Perlin. Challenges using head-mounted displays in shared and social spaces. In Extended Abstracts of the 2019 CHI Conference on Human Factors in Computing Systems, CHI EA '19, p. 1-8. Association for Computing Machinery, New York, NY, USA, 2019. doi: 10.1145/
3290607.3299028

[17] J. Gugenheimer, M. McGill, S. Huron, C. Mai, J. Williamson, and M. Nebeling. Exploring potentially abusive ethical, social and political implications of mixed reality research in hci. In Extended Abstracts of the 2020 CHI Conference on Human Factors in Computing Systems, CHI EA '20, p. 1-8. Association for Computing Machinery, New York, NY, USA, 2020. doi: 10.1145/3334480.3375180

[18] J. Gugenheimer, E. Stemasov, J. Frommel, and E. Rukzio. Sharevr: Enabling co-located experiences for virtual reality between hmd and non-hmd users. CHI '17, p. 4021-4033. Association for Computing Machinery, New York, NY, USA, 2017. doi: 10.1145/3025453. 3025683

[19] J. Gugenheimer, E. Stemasov, H. Sareen, and E. Rukzio. Facedisplay: Towards asymmetric multi-user interaction for nomadic virtual reality. In Proceedings of the 2018 CHI Conference on Human Factors in Computing Systems, CHI '18, p. 1-13. Association for Computing Machinery, New York, NY, USA, 2018. doi: 10.1145/3173574. 3173628

[20] I. Hirskyj-Douglas and A. Lucero. On the Internet, Nobody Knows You're a Dog... Unless You're Another Dog, p. 1-12. Association for Computing Machinery, New York, NY, USA, 2019.

[21] H. Ibayashi, Y. Sugiura, D. Sakamoto, N. Miyata, M. Tada, T. Okuma, T. Kurata, M. Mochimaru, and T. Igarashi. Dollhouse vr: A multiview, multi-user collaborative design workspace with vr technology. In SIGGRAPH Asia 2015 Posters, SA '15. Association for Computing Machinery, New York, NY, USA, 2015. doi: 10.1145/2820926. 2820948

[22] P. Jansen, F. Fischbach, J. Gugenheimer, E. Stemasov, J. Frommel, and E. Rukzio. Share: Enabling co-located asymmetric multi-user interaction for augmented reality head-mounted displays. In Proceedings of the 33rd Annual ACM Symposium on User Interface Software and Technology, UIST '20, p. 459-471. Association for Computing Machinery, New York, NY, USA, 2020. doi: 10.1145/3379337.3415843

[23] A. K. Karlson, A. B. Brush, and S. Schechter. Can i borrow your phone? understanding concerns when sharing mobile phones. In Proceedings of the SIGCHI Conference on Human Factors in Computing Systems, CHI '09, p. 1647-1650. Association for Computing Machinery, New York, NY, USA, 2009. doi: 10.1145/1518701.1518953

[24] C. Mai and M. Khamis. Public hmds: Modeling and understanding user behavior around public head-mounted displays. In Proceedings of the 7th ACM International Symposium on Pervasive Displays, PerDis '18. Association for Computing Machinery, New York, NY, USA, 2018. doi: $10.1145 / 3205873.3205879$

[25] C. Mai, A. Knittel, and H. Hußmann. Frontal screens on head-mounted displays to increase awareness of the hmd users' state in mixed presence collaboration. arXiv preprint arXiv:1905.06102, 2019.

[26] C. Mai, L. Rambold, and M. Khamis. Transparenthmd: Revealing the hmd user's face to bystanders. In Proceedings of the 16th International Conference on Mobile and Ubiquitous Multimedia, MUM '17, p. 515-520. Association for Computing Machinery, New York, NY, USA, 2017. doi: $10.1145 / 3152832.3157813$

[27] C. Mai, T. Wiltzius, F. Alt, and H. Hußmann. Feeling alone in public: Investigating the influence of spatial layout on users' vr experience. In Proceedings of the 10th Nordic Conference on Human-Computer Interaction, NordiCHI '18, p. 286-298. Association for Computing Machinery, New York, NY, USA, 2018. doi: 10.1145/3240167.3240200

[28] D. Marques, T. Guerreiro, and L. Carriço. Measuring snooping behavior with surveys: It's how you ask it. In CHI'14 Extended Abstracts on Human Factors in Computing Systems, CHI EA '14, p. 2479-2484. Association for Computing Machinery, New York, NY, USA, 2014. doi: $10.1145 / 2559206.2581240$

[29] D. Marques, I. Muslukhov, T. Guerreiro, L. Carriço, and K. Beznosov. Snooping on mobile phones: Prevalence and trends. In Twelfth Symposium on Usable Privacy and Security (SOUPS 2016), pp. 159-174. USENIX Association, Denver, CO, June 2016.

[30] F. Mathis and M. Khamis. Privacy, security and safety concerns of using hmds in public and semi-public spaces. Accessed: 2020-09-01.

[31] F. Mathis, J. Williamson, K. Vaniea, and M. Khamis. Rubikauth: fas and secure authentication in virtual reality. In Extended Abstracts of the 2020 CHI Conference on Human Factors in Computing Systems, 
pp. 1-9, 2020.

[32] F. Mathis, J. H. Williamson, K. Vaniea, and M. Khamis. Fast and secure authentication in virtual reality using coordinated $3 \mathrm{~d}$ manipulation and pointing. ACM Trans. Comput.-Hum. Interact., 28(1), Jan. 2021. doi: $10.1145 / 3428121$

[33] F. Mathis, X. Zhang, J. O'Hagan, D. Medeiros, P. Saeghe, M. McGill, S. Brewster, and M. Khamis. Remote xr studies: The golden future of hci research? 2021.

[34] M. McGill, D. Boland, R. Murray-Smith, and S. Brewster. A dose of reality: Overcoming usability challenges in vr head-mounted displays. In Proceedings of the 33rd Annual ACM Conference on Human Factors in Computing Systems, CHI '15, pp. 2143-2152. ACM, New York, NY, USA, 2015. doi: 10.1145/2702123.2702382

[35] M. McGill, J. Gugenheimer, and E. Freeman. A quest for co-located mixed reality: Aligning and assessing slam tracking for same-space multi-user experiences. In 26th ACM Symposium on Virtual Reality Software and Technology, VRST '20. Association for Computing Machinery, New York, NY, USA, 2020. doi: 10.1145/3385956.3418968

[36] D. Medeiros, R. d. Anjos, N. Pantidi, K. Huang, M. Sousa, C. Anslow, and J. Jorge. Promoting reality awareness in virtual reality through proxemics. In 2021 IEEE Virtual Reality and $3 D$ User Interfaces (VR), pp. 21-30, 2021. doi: 10.1109/VR50410.2021.00022

[37] Microsoft. Windows mixed reality flashlight. Software, October 2018. https://docs.microsoft.com/en-us/windows/mixed-reality/releasenotes-october-2018.

[38] Oculus. Oculus guardian. https://developer.oculus.com/ documentation/native/pc/dg-guardian-system/, May 2019. Accessed: 2020-09-01.

[39] J. O'Hagan, M. Khamis, and J. R. Williamson. Surveying consumer understanding sentiment of vr. In Proceedings of the International Workshop on Immersive Mixed and Virtual Environment Systems (MMVE '21), MMVE '21, p. 14-20. Association for Computing Machinery, New York, NY, USA, 2021. doi: 10.1145/3458307.3460965

[40] J. O'Hagan and J. R. Williamson. Reality aware vr headsets. In Proceedings of the 9TH ACM International Symposium on Pervasive Displays, PerDis '20, p. 9-17. Association for Computing Machinery, New York, NY, USA, 2020. doi: 10.1145/3393712.3395334

[41] J. O'Hagan, J. R. Williamson, and M. Khamis. Bystander interruption of vr users. In Proceedings of the 9TH ACM International Symposium on Pervasive Displays, PerDis '20, p. 19-27. Association for Computing Machinery, New York, NY, USA, 2020. doi: 10.1145/3393712. 3395339

[42] D. L. Paulhus, S. Vazire, et al. The self-report method. Handbook of research methods in personality psychology, 1(2007):224-239, 2007.

[43] PFMA. Families with pets 2019. https://www.pfma.org.uk/ families-with-pets-2019, April 2019. Accessed: 2020-05-27.

[44] T. Piumsomboon, Y. Lee, G. Lee, and M. Billinghurst. Covar: A collaborative virtual and augmented reality system for remote collaboration. In SIGGRAPH Asia 2017 Emerging Technologies, SA '17. Association for Computing Machinery, New York, NY, USA, 2017. doi: 10. $1145 / 3132818.3132822$

[45] R. Rzayev, S. Mayer, C. Krauter, and N. Henze. Notification in vr: The effect of notification placement, task and environment. In Proceedings of the Annual Symposium on Computer-Human Interaction in Play, CHI PLAY '19, p. 199-211. Association for Computing Machinery, New York, NY, USA, 2019. doi: 10.1145/3311350.3347190

[46] A. Scavarelli and R. J. Teather. Vr collide! comparing collisionavoidance methods between co-located virtual reality users. In Proceedings of the 2017 CHI Conference Extended Abstracts on Human Factors in Computing Systems, CHI EA '17, p. 2915-2921. Association for Computing Machinery, New York, NY, USA, 2017. doi: 10. 1145/3027063.3053180

[47] V. Schwind, J. Reinhardt, R. Rzayev, N. Henze, and K. Wolf. Virtual reality on the go? a study on social acceptance of vr glasses. MobileHCI '18, p. 111-118. Association for Computing Machinery, New York, NY, USA, 2018. doi: 10.1145/3236112.3236127

[48] A. L. Simeone. The vr motion tracker: visualising movement of nonparticipants in desktop virtual reality experiences. In 2016 IEEE 2nd Workshop on Everyday Virtual Reality (WEVR), pp. 1-4, 2016. doi: 10. 1109/WEVR.2016.7859535
[49] A. L. Simeone, E. Velloso, and H. Gellersen. Substitutional reality: Using the physical environment to design virtual reality experiences. In Proceedings of the 33rd Annual ACM Conference on Human Factors in Computing Systems, CHI '15, p. 3307-3316. Association for Computing Machinery, New York, NY, USA, 2015. doi: 10.1145/2702123. 2702389

[50] S. Thanyadit, P. Punpongsanon, and T.-C. Pong. Observar: Visualization system for observing virtual reality users using augmented reality. In 2019 IEEE International Symposium on Mixed and Augmented Reality (ISMAR), pp. 258-268, 2019. doi: 10.1109/ISMAR. 2019.00023

[51] R. Tourangeau and T. Yan. Sensitive questions in surveys. Psychological bulletin, 133(5):859, 2007.

[52] Valve. Valve chaperone. https://support.steampowered.com/ kb_article.php?ref=6281-TOKV-4722, April 2016. Accessed: 2020-09-01.

[53] A. Vinciarelli, M. Pantic, and H. Bourlard. Social signal processing: Survey of an emerging domain. Image and Vision Computing, 27(12):1743-1759, 2009. Visual and multimodal analysis of human spontaneous behaviour:. doi: 10.1016/j.imavis.2008.11.007

[54] J. von Willich, M. Funk, F. Müller, K. Marky, J. Riemann, and M. Mühlhäuser. You invaded my tracking space! using augmented virtuality for spotting passersby in room-scale virtual reality. In Proceedings of the 2019 on Designing Interactive Systems Conference, DIS '19, pp. 487-496. ACM, New York, NY, USA, 2019. doi: 10. $1145 / 3322276.3322334$

[55] J. R. Williamson, M. McGill, and K. Outram. PlaneVR: Social Acceptability of Virtual Reality for Aeroplane Passengers, p. 1-14. Association for Computing Machinery, New York, NY, USA, 2019.

[56] L. K. Woolsey. The critical incident technique: An innovative qualitative method of research. Canadian Journal of Counselling and Psychotherapy, 20(4), 1986.

[57] K.-T. Yang, C.-H. Wang, and L. Chan. Sharespace: Facilitating shared use of the physical space by both vr head-mounted display and external users. In Proceedings of the 31st Annual ACM Symposium on User Interface Software and Technology, UIST '18, pp. 499-509. ACM, New York, NY, USA, 2018. doi: 10.1145/3242587.3242630 